\title{
Higher Serum Ferritin in Tibetan and Han Populations with Diabetes Living on the Tibetan Plateau
}

\author{
Zhenzhong Bai ${ }^{1,2,3 *}$, Chengyu Zhao ${ }^{2,3 *}$, Shou Liu ${ }^{1}$, Renjie Feng ${ }^{4}$, Sen Cui ${ }^{3}$, Ri-li Ge ${ }^{1 *}$, Donald McClain ${ }^{2,5 *}$ \\ ${ }^{1}$ Research Center for High Altitude Medicine, Qinghai University Medical School, 810001 Qinghai Xining, the People's Republic of China \\ ${ }^{2}$ Center of Obesity and Diabetes, University of Wake Forest, School of Medicine, Winston Salem, North Carolina 27104, USA \\ ${ }^{3}$ Affiliated Hospital of Qinghai University, Qinghai University, 810001 Qinghai Xining, the People's Republic of China \\ ${ }^{4}$ Department of Public Health, Wuhan University, Wuhan, Hubei, the People's Republic of China \\ ${ }^{5}$ Veterans Affairs Research Service, Salisbury, North Carolina 28144, USA \\ "ZB and CZ contributed equally \\ *Correspondence to:
}

Donald McClain, Center of Obesity and Diabetes, University of Wake Forest, School of Medicine, Winston Salem, North Carolina 27104, USA; E-mail: dmcclain@ wakehealth.edu

Ri-Li Ge, Research Center for High Altitude Medicine, Qinghai University, Xining 810001, China; E-mail: geriligao@hotmail.com

Received: December 14, 2017; Accepted: December 21, 2017; Published: December 23, 2017;

\begin{abstract}
Objective: Tissue iron has emerged as a significant risk factor for diabetes. Pathways that sense and regulate iron and oxygen interact, but few studies examined the interactions of hypoxia and iron in determining diabetes risk in human populations. Accordingly, metabolic phenotyping with analysis of iron homeostasis in both Tibetan and Han Chinese living at 2300-3900m altitudes were conducted.
\end{abstract}

Research design and methods: Data were collected on Tibetan and Han Chinese living at intermediate altitudes. Iron homeostatic and metabolic parameters including homeostasis model assessments (HOMA), hemoglobin A1c, serum ferritin and transferrin saturation were determined.

Results: Serum ferritin is higher in both Tibetan groups compared to the respective Han groups, and higher in each diabetic group compared to nondiabetics of the same ethnicity. Serum iron and transferrin saturation were also higher in the Tibetan diabetics than the Tibetan non-diabetics. Serum iron was significantly correlated with ferritin levels in the four combined groups $\left(\mathrm{r}^{2}=0.07313, \mathrm{p}<0.05\right)$ and even stronger in the Tibetan diabetic group $\left(r^{2}=0.2702, p<0.05\right)$. HOMA- $\beta$ was negatively correlated with ferritin in the Tibetan combined groups $\left(r^{2}=0.020, p<0.05\right)$, and HOMA-IR tended to be positively correlated with ferritin $\left(\mathrm{r}^{2}=0.018, \mathrm{p}<0.05\right)$.

Conclusion: Iron parameters differ both between Han and Tibetans and between diabetics and nondiabetics of both populations. High ferritin, which in these cohorts reflects iron status, is a risk factor for diabetes in both groups, although how iron status relates to the diabetes phenotype differs between the two groups, possibly related to their differing histories of adaptation to high altitude.

Keywords: Iron; Ferritin; Diabetes; Tibetan Adaptation

\section{Key Messages}

- The study presents evidence of the relationship between serum ferritin, a measure of tissue iron stores that has been linked to diabetes risk in other populations, and diabetes in Tibetan and Han populations living at altitudes of 2300 m-3900 m.

- The study also presents associations of glucose metabolic phenotypes (homeostasis model assessments, HOMA) in those with diabetes.

- Due to the relatively low prevalence of diabetes in Tibetan populations, the sample size was constrained

- This study is cross-sectional and therefore, future prospective and follow-up studies to reveal the interactions analysis of iron metabolism and diabetes in Tibetans and Han populations are indicated.

- $\quad$ The ethnic background of the subjects is by self-report

\section{Introduction}

Many factors contribute to the risk of diabetes, including genetics, ethnicity, altitude, and diet [1]. Tissue iron has emerged as an additional significant risk factor for diabetes and has been shown to play an important role in metabolic regulation $[2,3]$. The initial association between diabetes risk and iron emerged from studies of heritable diseases of iron overload such as hereditary hemochromatosis $(\mathrm{HH})$ [4]. More recent studies demonstrate that the relation between iron and diabetes also exists in the general population and across the broad range of "normal" iron, and an associations between iron and diabetes have been established in Caucasians [3,5], persons of African descent [6], and several Asian populations [7-11,12]. The mechanism by which 
iron confers diabetes risk, however, is not established in all of these populations, and the interactions of genetics, diet, and geography with the degree of risk are also unknown.

The interaction of iron and altitudes particularly interesting because residence at higher altitudes is inversely associated with diabetes, but hypoxia responsive pathways also increase iron absorption to allow increased erythropoiesis [13]. Tibetans have successfully adapted to reside at altitudes of up to $5000 \mathrm{~m}[14,15]$ and it has been reported that there is a reduced prevalence of diabetes and metabolic syndrome in Tibetans [16]. The genetic determinants of that adaptation include changes in the hypoxia sensing pathway [15]. One characterized genetic mechanism, for example, is a gain of function mutation that confers increased oxygen affinity to the proline hydroxylase that would normally be inactivated by hypoxia, thus facilitating stabilization of hypoxia inducible factors [17]. The adaptation was hypothesized to protect Tibetans from the induction of harmful levels of polycythemia. The mutation, which partially disables the hypoxia response, would also be expected to render Tibetans less sensitive to induction of the diabetes-protective hypoxia response to altitude. Because the hypoxia sensing pathway exerts major effects on multiple metabolic pathways and senses both tissue iron and oxygen levels [18], it is likely that altitude and iron interact to affect metabolism and diabetes risk, and that these interactions may differ in Tibetans. Interactions between iron and hypoxia have been studied in animal models and shown to affect hepatic glucose production [19], for example, a major factor in diabetes, but their interaction in human populations has not been examined.

In order to begin to address these issues, we have examined metabolic phenotypes and measures of iron homeostasis in Tibetan and Han Chinese with and without diabetes, living at altitudes of 2300-3900 m. The aim of this study was to identify how iron and high altitude hypoxia interact in a population of Tibetan highlanders genetically adapted to high altitude, compared to the non-adapted Han Chinese.

\section{Participants and Methods}

\section{Participants}

This cross-sectional study included one hundred Tibetan residents of an urban centre (Yushu, 3300-3900m altitude), with and without diabetes, randomly selected from a population receiving routine health screening, at Qinghai University Affiliated Hospital. One hundred Han Chinese individuals, with and without diabetes, matched for age and sex, were selected from a clinic-based population, at the Qinghai University Affiliated Hospital, in Xining (2300m). All the participants were chosen between September 2013 and September 2016.

\section{Data collection and measurement}

Clinical characteristics including sex, age, body mass index (BMI), and medical history were recorded. Height and weight were measured according to a standardized protocol and technique, with participants wearing no shoes. BMI was calculated as weight $(\mathrm{kg}) / \mathrm{height}\left(\mathrm{m}^{2}\right)$. Blood pressure was measured by trained professionals, using an electronic sphygmomanometer (OMRON HEM-7200), and each participant rested for at least $20 \mathrm{~min}$ before measurements were taken.
Health screening included the measurement of fasting glucose and glycated haemoglobin (HbAlc) values. Blood samples were drawn from participants' antecubital veins for measuring fasting blood glucose (FBG), triglycerides (TGs), total cholesterol (TC), lowdensity lipoprotein cholesterol (LDL-C) and high-density lipoprotein cholesterol (HDL-C) levels. From this information, de-identified participants of each ethnicity (Tibetan and Han Chinese), with no known history of diabetes and who had not taken any insulin or related drugs, were chosen. From among them, 100 individuals of each ethnicity who were newly diagnosed as having diabetes, based on fasting glucose $(\geq 126 \mathrm{mg} / \mathrm{dl})$ or HbAlc $(\geq 6.5 \%)$ criteria, or who were nondiabetic, were randomly chosen. Individuals with known hematologic disorders, previously diagnosed diabetes, a history of recent (within 1 year) blood transfusion, and malignancy were excluded. In the case of those individuals, the blood samples drawn for health screening were used for the determination of levels of serum insulin and lipids, and iron parameters. The study was approved by the Ethnic Committee of Qinghai University, and the Institutional Review Board of Wake Forest University. Extra clinical samples were used for the additional testing of iron parameters.

\section{Laboratory studies}

Fasting blood glucose (FBG), fasting serum insulin, ferritin and serum iron, TG, LDL, HDL, total cholesterol (TH), and HbAlc levels were analysed by latex agglutination immunoassay (DCA $2000 \mathrm{HbA1c}$, Siemens Healthcare Co Ltd, Munich, Germany). These parameters were measured by standard techniques used in the clinical chemistry laboratories at the Qinghai University Affiliated Hospital. HOMA-IR and $\beta$-cell function (HOMA- $\beta$ ) (\%) values were calculated as [FPG $(\mathrm{mg} / \mathrm{dl}) \times \operatorname{IRI}(\mu \mathrm{U} / \mathrm{ml})] / 405$ and [IRI $(\mu \mathrm{U} / \mathrm{ml}) \times 360] /[\mathrm{FPG}$ (mg/dl)-63], respectively.

Iron metabolic parameters were measured using an automated time-resolved immunofluorometric assay (Finland) and the reference values were: Serum iron, Male 11.6-31.7 $\mu \mathrm{mol} / \mathrm{L}$; Female 9.0-30.4 $\mu \mathrm{mol} / \mathrm{L}$, Total Iron Binding Capacity: 45-66 $\mu \mathrm{mol} / \mathrm{L}$, Transferring saturation, Male 20-50\%; Female 15-50\%, and Serum ferritin, Male 20-240 ng/mL; Female, $10-100$ ng/mL)

\section{Statistical analysis}

Continuous variables that followed a skewed distribution were transformed to normal distribution by natural log or square root transformation. Using a Students' $t$-test or Chi-square, the distribution of clinical and laboratory measurements was tested between individuals with and without diabetes, in the Han Chinese and Tibetan groups, respectively. Pearson and Spearman correlations were used to assess the correlations between ferritin levels, iron concentrations and other variables, in the two groups. Analyses were performed using SPSS 14.0 for Windows software package (SPSS, Chicago, IL). $P$ values $<0.05$ were considered statistically significant.

\section{Results}

\section{Characteristics of the Tibetan and Han Chinese study populations}

Tibetan residents with diabetes $(\mathrm{n}=100)$ and Han Chinese with diabetes $(n=100)$ were matched to 100 subjects of each group 
without diabetes, with respect to age, gender, body mass index, total hemoglobin and blood pressure (Table 1). As expected, each diabetic group had higher fasting glucose values than their respective nondiabetic group. HbA1c was not determined in those with FBG less than $126 \mathrm{mg} / \mathrm{dL}$. In the Tibetan diabetes group, surprisingly lower triglyceride levels, and lower HDL and higher LDL were noted compared to the non-diabetic group. LDL cholesterol concentrations were also higher the non-diabetic Han compared to the nondiabetic Tibetans (Table 1).

Iron parameters differ between Han Chinese and Tibetan populations, and between those with and without diabetes, and we identified a unique association between diabetes risk and the iron indices, in Tibetans. Because of our study population, which included Tibetans and Han Chinese individuals, both of whom reside in intermediate altitudes of 2300-3900 m, we examined the interrelations between serum iron-related parameters, ethnicity, and diabetes status, respectively. The ferritin level was higher in the diabetes group compared to the non-diabetes group, within the same ethnicity $(\mathrm{p}<0.01$, Figure $1 \mathrm{~A})$. Our hypothesis is that, in the non-diabetes groups there should no difference in the ferritin level; however, it was observed that the ferritin level was higher in both Tibetan diabetes and non-diabetes groups, compared to the respective Han Chinese groups $(\mathrm{p}<0.01)$; this indicates that Tibetan populations showed specialized tissue iron storage.

Next, we assessed both $\beta$-cell function and insulin resistance, according to the homeostasis model assessment (HOMA- $\beta$ and HOMA-IR). Insulin values were only available in the diabetes groups. The HOMA-IR values were greater than 2 in both the diabetes groups (Table 2); this was consistent with insulin resistance [19]. The HOMAIR and HOMA- $\beta$ values did not differ between the Han Chinese and Tibetan populations. The HOMA- $\beta$ value was negatively correlated with ferritin, in both groups $\left(\mathrm{r}^{2}=-0.02, \mathrm{p}=0.04\right)$, and the HOMA-IR value tended to be positively correlated with ferritin $\left(r^{2}=0.02, p=0.05\right)$. Within each ethnicity, the same trends held, but did not reach significance in the smaller groups (Table 2).

Table 1 Distribution of variables in Tibetan and Han Chinese populations with and without diabetes. Results are presented as median (interquartile ranges).

\begin{tabular}{|c|c|c|c|c|}
\hline \multirow[t]{2}{*}{ Variable } & \multicolumn{2}{|c|}{ Tibetan } & \multicolumn{2}{|c|}{ Han Chinese } \\
\hline & $\begin{array}{l}\text { Diabetes group } \\
\qquad \mathbf{n}=100\end{array}$ & $\begin{array}{l}\text { Non-diabetes group } \\
\qquad \mathbf{n}=100\end{array}$ & $\begin{array}{l}\text { Diabetes group } \\
\qquad n=100\end{array}$ & $\begin{array}{l}\text { Non-diabetes group } \\
\qquad n=100\end{array}$ \\
\hline Age(years) & $50(44 \sim 59.75)$ & $50(45 \sim 58.75)$ & $52(46 \sim 58)$ & $54(48 \sim 58)$ \\
\hline Gender (male\%) & $54 \%$ & $52 \%$ & $49 \%$ & $55 \%$ \\
\hline BMI $\left(\mathbf{k g} / \mathbf{m}^{2}\right)$ & $24.08(22.53 \sim 26.23)$ & $24.18(21.76 \sim 26.5)$ & $24.22(22.2 \sim 26.2)$ & 24.15(21.84 25.95) \\
\hline Haemoglobin (g/L) & $160(150 \sim 172)$ & 163.02(146.97 172.09) & $163(150 \sim 172)$ & $160.34(144.49 \sim 173.58)$ \\
\hline Diastolic Pressure (mmHg) & $80(80 \sim 88)$ & $80(80 \sim 84)$ & $80(76 \sim 89)$ & $80(78.25 \sim 85.75)$ \\
\hline Systolic Pressure(mmHg) & $124(120 \sim 140)$ & $120(120 \sim 130)$ & $120(120 \sim 130)$ & $120(119.25 \sim 137.25)$ \\
\hline Fasting Glucose(mmol/L) & $9.36(8 \sim 10.94)$ & $4.59(4.3 \sim 5)$ & $8.15(6.8 \sim 11.39)$ & $4.6(4.3 \sim 5.06)$ \\
\hline Glycated Haemoglobin (\%) & $10.25(8.55 \sim 11.88)$ & - & $8.8(7.05 \sim 10.2) *$ & - \\
\hline Triglyceride(mmol/L) & $1.68(1.32 \sim 2.33)$ & $1.71(1.38 \sim 2.35)^{\Lambda}$ & $1.73(1.32 \sim 2.32)^{*}$ & $1.76(1.36 \sim 2)$ \\
\hline Cholesterol(mmol/L) & $4.58(4 \sim 4.93)$ & $4.57(3.88 \sim 5.09)$ & $4.52(3.96 \sim 5)$ & $4.26(3.75 \sim 5)$ \\
\hline HDL(mmol/L) & $0.97(0.81 \sim 1.13)$ & $1.03(0.85 \sim 1.19)^{\Lambda}$ & $1.03(0.88 \sim 1.19)^{*}$ & $1.08(0.88 \sim 1.26)$ \\
\hline $\mathrm{LDL}(\mathrm{mmol} / \mathrm{L})$ & $3.01(2.25 \sim 3.63)$ & $2.44(2.03 \sim 2.94)^{\Lambda}$ & $2.58(2.1 \sim 3.28)^{*}$ & $2.57(2 \sim 3)$ \\
\hline \multicolumn{5}{|l|}{ Iron Metabolism } \\
\hline $\operatorname{Iron}(\mathrm{umol} / \mathrm{L})$ & $20(16.4 \sim 24.9)$ & $15.6(12 \sim 20.58)^{\Delta}$ & $16.9(12.85 \sim 22)^{*}$ & $15.75(12.9 \sim 18.83)$ \\
\hline Ferritin (ng/ml) & $613.89(457.07 \sim 812.75)$ & $279.85(231.32 \sim 359.56)^{\Delta}$ & $234.66(173.68 \sim 325.25)^{*}$ & $78(59.45 \sim 98.63)^{+}$ \\
\hline TIBC(umol/L) & 29.3(23.1 35) & $48.6(43.13 \sim 57.08)^{\Delta}$ & $38(33.05 \sim 43.55)^{*}$ & $51.65(47.43 \sim 57.4)^{+}$ \\
\hline TSAT(\%) & $0.7(0.5 \sim 0.98)$ & $0.33(0.25 \sim 0.39)^{\Delta}$ & $0.46(0.31 \sim 0.64)^{*}$ & $0.30(0.25 \sim 0.35)^{+}$ \\
\hline
\end{tabular}

Notes: * for Tibetan diabetes group vs Han Chinese diabetes group with a significant difference of $\mathrm{p}<0.05 ;{ }^{\wedge}$ for Tibetan diabetes group vs Tibetan non-diabetes group with a significant difference of $\mathrm{p}<0.05 ;{ }^{+}$for Tibetan non-diabetes group vs Han Chinese diabetes group with a significant difference of $\mathrm{p}<0.05$

Abbreviations: LDL, low-density lipoprotein; HDL, high-density-lipoprotein; TIBC, total iron binding capacity; TSAT, transferrin saturation

Table 2. Distribution of HOMA values in Tibetan and Han Chinese diabetes groups. Results are presented as median (interquartile ranges)

\begin{tabular}{|l|l|l|c|l|}
\hline Variables & $\begin{array}{c}\text { Tibetans with diabetes } \\
\mathbf{N = 1 0 0}\end{array}$ & $\begin{array}{c}\text { Han Chinese with } \\
\text { diabetes } \\
\mathbf{N = 1 0 0}\end{array}$ & $\boldsymbol{Z}$ & $\boldsymbol{p}$ \\
\hline HOMA-IR & $29.35(22.25 \sim 34)$ & $25.64(17.02 \sim 46.79)$ & -0.293 & 0.77 \\
HOMA- $\beta$ & $2.35(1.52 \sim 4.04)$ & $2.44(1.49 \sim 3.59)$ & -0.684 & 0.494 \\
\hline
\end{tabular}

Abbreviations: HOMA, homeostasis model assessment; HOMA-IR, homeostasis model assessment insulin resistance 

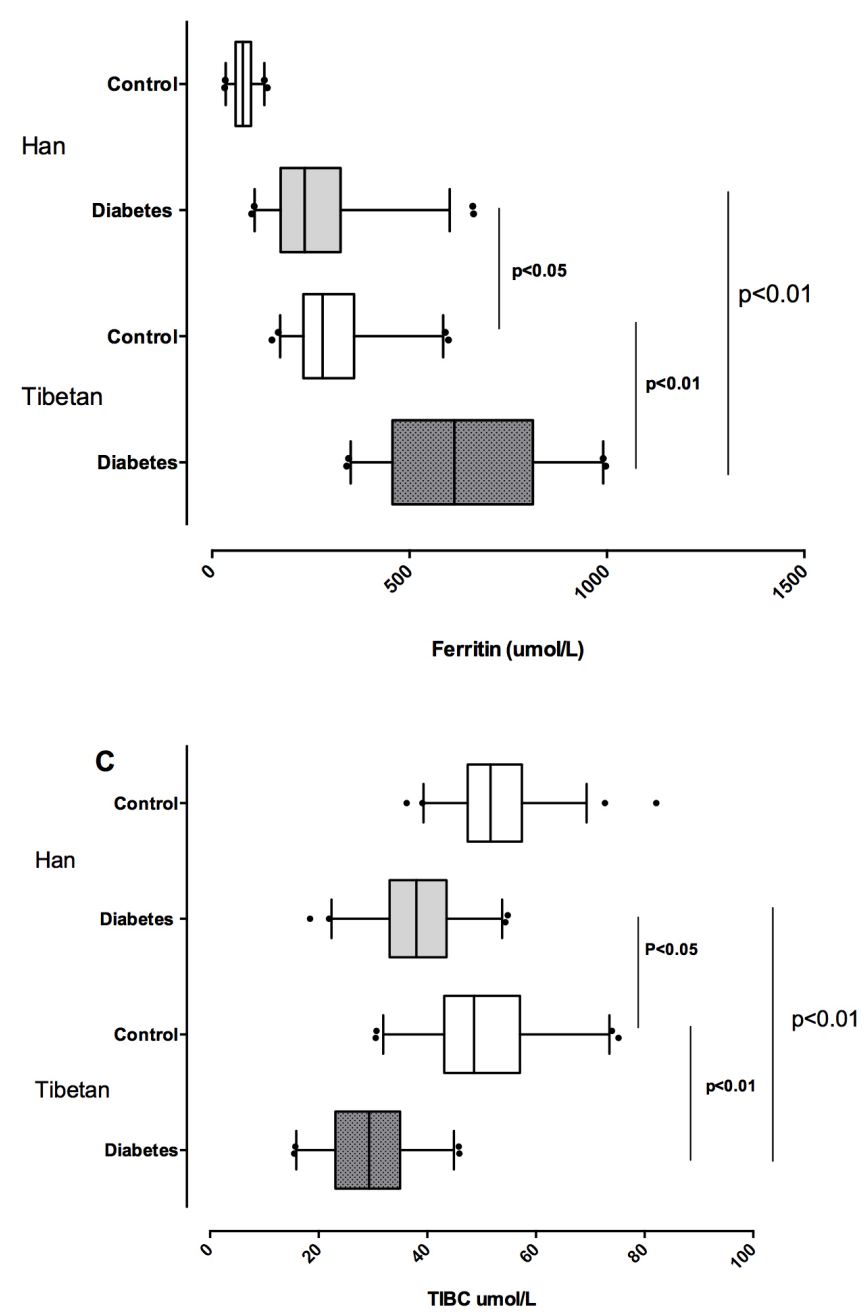

B
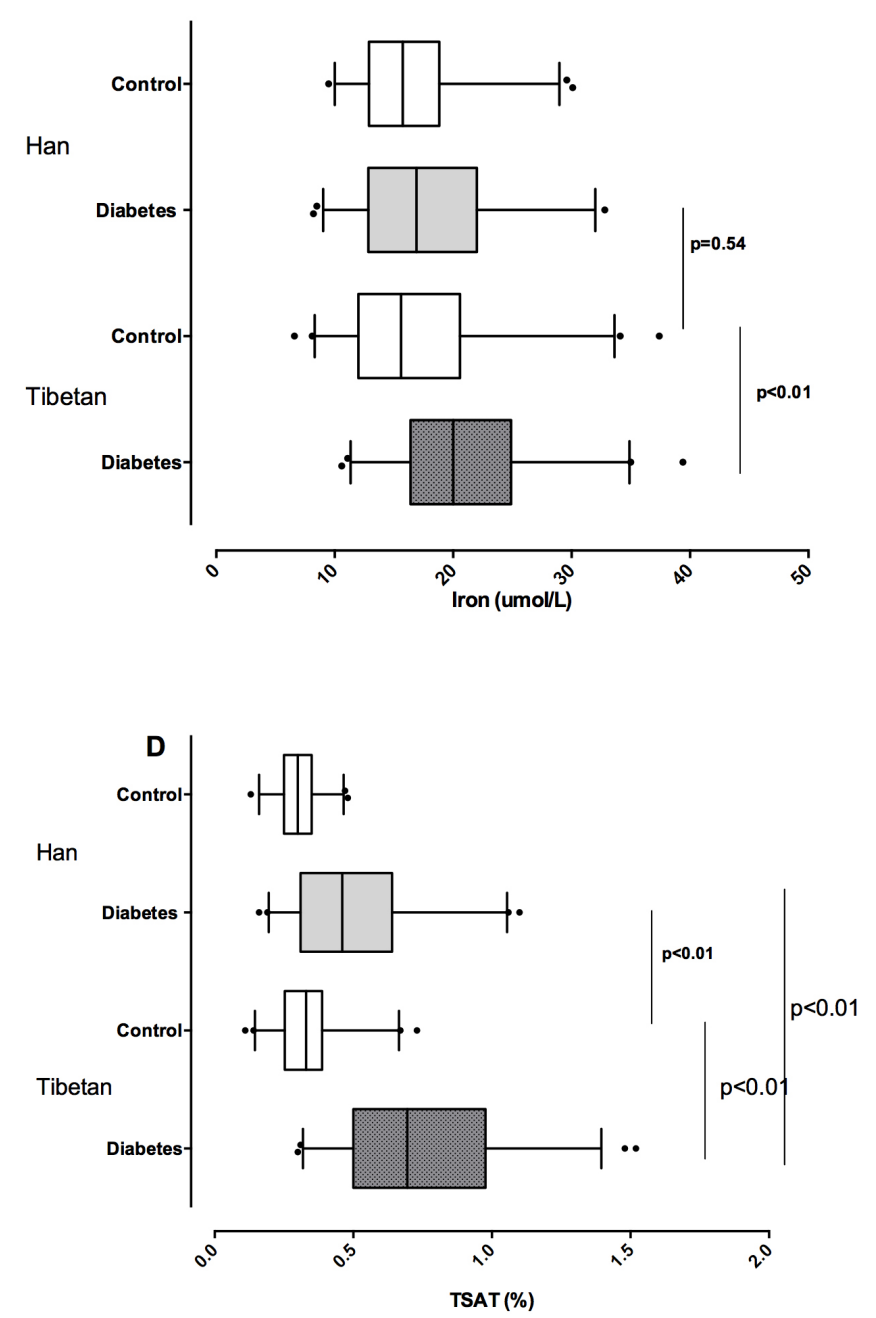

Figure 1. Iron metabolic values in Tibetan and Han Chinese groups with and without diabetes, in terms of A) Serum ferritin level comparisons; B) Serum iron level comparisons; C) Total iron binding capacity comparisons; and D) Transferrin saturation ratio comparisons.

*dot spot indicates the 5-95 percentile of the values.

Serum ferritin reflects the iron status in the study group, while other iron parameters are also associated with diabetes

After, we examined serum iron levels in the four groups. Like in the case of ferritin, the serum iron level was higher in Tibetans with diabetes than Tibetan without it; in addition, it was higher in Tibetan participants with diabetes than Han Chinese participants with diabetes ( $\mathrm{p}<0.01$ for both comparisons, Figure $1 \mathrm{~B}$ ), although the trend among Han Chinese participants with diabetes was only higher than those without diabetes in the same population $(\mathrm{p}<0.05)$. The same was true of transferrin saturation ( $\mathrm{p}<0.01$, Figure 1D). As further evidence that ferritin reflects iron status rather than inflammation, in our study, the serum iron and ferritin levels were significantly correlated in the four groups $\left(r^{2}=0.07, p<0.01\right.$, Figure 2$)$. The correlation was even stronger in the Tibetan diabetes group $\left(\mathrm{r}^{2}=0.27, \mathrm{p}<0.01\right)$, but was not significant in the other groups, taken individually.

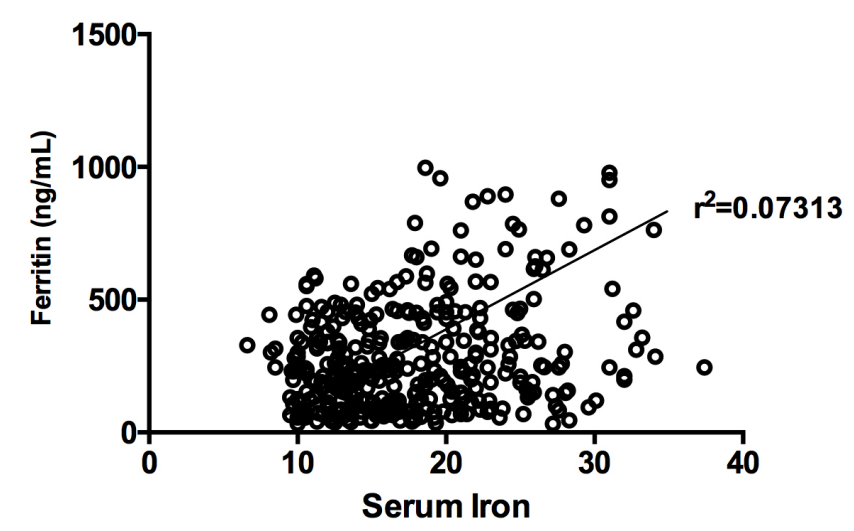

Figure 2. Correlation of serum iron and ferritin levels in Tibetan and Han Chinese populations, with and without diabetes. Scatter-plot diagram. 


\section{Higher ferritin levels identified as a new pattern of diabetes risk in Tibetans}

Insulin resistance and decreased $\beta$-cell function can also result from as well as cause poor plasma glucose stability and hyperglycemia [20]. Therefore, we determined if glucose stability influenced the HOMA- $\beta$ and HOMA-IR values. The associations of HbAlc levels with the HOMA-IR and HOMA- $\beta$ values were assessed, respectively. However, HbA1c levels were not found to be correlated with HOMAIR $(\mathrm{p}=0.95)$, HOMA- $\beta$ or ferritin $(\mathrm{p}=0.63)$, in the Tibetan diabetes group. In the Han Chinese diabetes group, however, HbAlcwas significantly negatively correlated with ferritin $(\mathrm{p}=0.04)$ and the HOMA- $\beta(\mathrm{p}=0.04)$ value, and trended toward being negatively correlated with the HOMA-IR $(\mathrm{p}=0.11)$ value.

\section{Discussion}

\section{Iron and diabetes risk in Tibetan and Han Chinese}

The connection between iron and diabetes have been the subject of recent reviews [3,4,21-23] and a meta-analysis [24-26]. Originally identified in subjects with hereditary iron overload such as occurs in hereditary hemochromatosis [27,28], the correlation between markers of tissue iron and diabetes has since been noted in numerous populations in the U.S., Europe [3] and elsewhere, including Korean [7], Chinese Han [8-11], African Americans [6,29], and Persian populations [30]. Our findings generally support these studies insofar as Tibetans diabetics have higher ferritin than nondiabetics, and Han diabetics have higher ferritin than Han nondiabetics. However, serum ferritin can rise with systemic inflammation as well as tissue iron, and phlebotomy studies have shown ferritin to be related to tissue iron, but imperfectly so [31]. In general, however, tissue iron, in particular liver iron, is the predominant determinant of serum ferritin levels [32], and ferritin correlates very well with liver iron by imaging criteria [33]. Even in the inflammatory condition of non-alcoholic steatohepatitis, ferritin remains are liable reporter of tissue iron as assessed by liver biopsy [34]. Further evidence that ferritin is reporting on iron status is the correlation of ferritin with serum iron. Thus, although we do not have direct measures of tissue iron content, the results overall support the finding in multiple populations of a correlation of iron with diabetes prevalence. Further support for this conclusion is the large amount of data from animal and human models that support causal connections between iron and diabetes, mediated both by $\beta$-cell failure and insulin resistance with the underlying mechanisms including oxidant stress and modulation of adipokines that affect glycemia $[29,31,35]$, and data from NHANES showing that ferritin reflects dietary iron intake rather than inflammation [5].

Our findings also indicate that ferritin remains associated with diabetes in residents of higher altitudes. This replicates a previously reported association of ferritin with diabetes in Han Chinese living at altitude [36]. Living at higher altitudes has been reported to be associated with lower diabetes risk [35] and likewise activation of hypoxia-sensing pathways has salutary effects on glucose homeostasis [37]. Thus, our study indicates that in both populations the association with iron is not erased by the beneficial effects of altitude, though we do not have diabetes incidence and prevalence data as a function of iron with which to examine their possible interaction.
We also show that Tibetans with and without diabetes have significantly higher ferritin levels and transferrin saturations than the Han population. The reasons for this are not clear. One possibility is diet, with the traditional Tibetan diet including substantial amounts of high-iron barley [1], and red yak meat; dietary surveys were not done in this study but would be useful in future studies aimed at explaining these parameters. Epidemiological investigations are needed to explain if the relative risk of diabetes for a given ferritin level is altered in Tibetans compared to Han. Interestingly, although the transferrin saturation is significantly higher in Tibetans with diabetes, which is accounted as much by low iron binding capacity as by higher serum iron, but the basis for this is not known. Finally, hypoxia also induces increased absorption of dietary iron to support polycythemia at altitude. Tibetans have genetically adapted to high altitude with unique haplotypes and mutations in genes of the hypoxia sensing pathways [13], and this may explain part of the differences between the Tibetans and Han in the current study, who are residing at similar altitudes.

\section{Mechanisms of iron-associated diabetes in the Han and Tibetans}

Further indication that the association of ferritin, iron and diabetes risk is complex is that if the relationship were simply one of high iron, and if the high ferritin values in Tibetans did simply reflect high iron, that might imply higher diabetes risk in that population, whereas epidemiologic studies suggest that Tibetans have a lower prevalence to diabetes [9]. A minority of the subjects in this study were overweight, with average BMI in all groups being $\sim 24$, consistent with the reported association of altitude with leanness [38], and that might be expected, like the altitude itself [1], to counter any degree of iron-associated risk that might also be present. The associations of ferritin with HOMA- $\beta$ and HOMA-IR were weaker than observed in the other populations cited in the Introduction, yet similar between Han and Tibetan groups. This, too, may be related to the dilution out of these effects by the altitude. The results suggest that altitude adds a new dimension to reported diabetes risk associations, and that further studies of diabetes prevalence in different populations living at high and low altitudes, with more detailed physiologic phenotyping and genotyping, may shed light on the pathogenesis of this disease.

\section{Conclusions, unanswered questions and implication}

To our knowledge, this is the first study to suggest that a special relationship exists between tissue iron storage and diabetes risk, in Tibetan populations. Furthermore, it is suggested that residing in high altitudes may impact the associations between the glucose metabolic phenotypes, and lead to more stabilised glucose levels and higher dependency on glucose for energy. In addition, the new patterns of iron homeostasis in Tibetans with urban lifestyles living at high altitudes might contribute to the special adaptations. Notably, since this study had a cross-sectional study design that involved a review of health record data, it was also impossible to obtain other data that may have been of interest, such as dietary histories, or further laboratory measures of other iron-related measures. Therefore, a future perspective and follow-up study to analyse the interactions of 
iron metabolism and diabetes, in Tibetan populations, is required. Most importantly, the genetic background of the study's participants was not identified. In addition, the major regulators of iron such as hepcidin and transferring were also not investigated, and both factors play emerged effects on iron homeostasis in human beings. The prevention and management of diabetes through dietary iron intake intervention should be a potential therapy target in the future.

\section{Contributors}

$\mathrm{ZB}$ and $\mathrm{DM}$ wrote the manuscript; $\mathrm{CZ}$ and $\mathrm{JZ}$ collected and supplied the data; SL with YZ, RF and DM analysed the data; SC with RG designed and coordinated the study. All authors reviewed and approved the final manuscript.

Acknowledgements: The authors thank all the participants, as well all those who provided support.

\section{Funding}

This research was supported in China, by the: 1 . National Program on Key Basic Research Project of China (No.2012CB518200) 2. National Natural Science Foundation of China (No. 31571231) 3. Science and Technology Department, International Cooperation Program of Qinghai Province (2015-HZ-807), and in the USA by: 4 . United States National Institutes of Health1R01 DK081842 (DAM) and 5UL1 TR001420-02 (DAM); 5. United States Department of Veterans Affairs 2I01 BX001140 (DAM).

\section{Competing interests: None.}

Ethics approval: The Institutional Ethnic Committee of Qinghai University and the Institutional Review Board of Wake Forest University approved the study.

Data sharing statement: The data that support the findings of this study can be obtained from the corresponding author upon reasonable request.

\section{References}

1. Wang Z, Dang S, Yan H (2010) Nutrient intakes of rural Tibetan mothers: a crosssectional survey. BMC Public Health 10: 801-801. [Crossref]

2. Swaminathan S, Fonseca VA, Alam MG, Shah SV (2007) The role of iron in diabetes and its complications. Diabetes Care 30: 1926-1933. [Crossref]

3. Simcox JA, McClain DA (2013) Iron and diabetes risk. Cell Metab 17: 329-341. [Crossref]

4. Fernández-Real JM, McClain D, Manco M (2015) Mechanisms linking glucose homeostasis and iron metabolism toward the onset and progression of type 2 diabetes. Diabetes Care 38(11): 2169-2176. [Crossref]

5. Ford ES, Cogswell ME (1999) Diabetes and serum ferritin concentration among U.S. adults. Diabetes Care 22(12): 1978. [Crossref]

6. Wilson JG, Lindquist JH, Grambow SC, Crook ED, Maher JF (2003) Potential role of increased iron stores in diabetes. American Journal of the Medical Sciences 325(6): p. 332-339. [Crossref]

7. Kim CH, Kim HK, Bae SJ, Park JY, Lee KU (2011) Association of elevated serum ferritin concentration with insulin resistance and impaired glucose metabolism in Korean men and women. Metabolism 60(3): 414-420. [Crossref]

8. Liu BW, Xuan XM, Liu JR, Li FN, Fu-Zai Yin (2015) The Relationship between Serum Ferritin and Insulin Resistance in Different Glucose Metabolism in Nonobese Han Adults. Int J Endocrin 642194

9. Jiang R, Manson JE, Meigs JB, Ma J, Rifai N, et al. (2004) Body iron stores in relation to risk of type 2 diabetes in apparently healthy women. JAMA 291: 711717. [Crossref]
10. Shi Z, Hu X, Yuan B, Pan X, Meyer HE (2006) Association Between Serum Ferritin, Hemoglobin, Iron Intake, and Diabetes in Adults in Jiangsu, China. Diabetes Care 29(8): p. 1878. [Crossref]

11. Luan de C, Li H, Li SJ, Zhao Z, Li X, Liu ZM (2008) Body Iron Stores and Dietary Iron Intake in Relation to Diabetes in Adults in North China. Diabetes Care 31(2): p. 285. [Crossref]

12. Jung CH, Lee MJ, Hwang JY, Jang JE, Leem J, et al. (2013) Elevated Serum Ferritin Level Is Associated with the Incident Type 2 Diabetes in Healthy Korean Men: A 4 Year Longitudinal Study. PLoS One 8(9): p. e75250. [Crossref]

13. Gassmann M, Muckenthaler MU (2015) Adaptation of iron requirement to hypoxic conditions at high altitude. J Appl Physiol (1985) 119: 1432-1440. [Crossref]

14. West JB (2012) Concise Clinical Review: High Altitude Medicine. Am J Resp Crit Care Med 186(12): p. 1229-1237. [Crossref]

15. Simonson TS, McClain DA, Jorde LB, Prchal JT (2012) Genetic determinants of Tibetan high-altitude adaptation. Hum Genet 131: 527-533. [Crossref]

16. Okumiya K, Sakamoto R, Ishimoto Y, Kimura Y, Fukutomi E, et al. (2016) Glucose intolerance associated with hypoxia in people living at high altitudes in the Tibetan highland. BMJ Open 6(2): p. e009728. [Crossref]

17. Lorenzo FR, Huff C, Myllymäki M, Olenchock B, Swierczek S, et al. (2014) A genetic mechanism for Tibetan high-altitude adaptation. Nat Genet 46: 951-956. [Crossref]

18. Semenza GL (2004) Hydroxylation of HIF-1: Oxygen Sensing at the Molecular Level. Physiology (Bethesda) 19(4): p. 176. [Crossref]

19. Rabasa-Lhoret R, Bastard JP, Jan V, Ducluzeau PH, Andreelli F, Guebre F, et al (2003) Modified Quantitative Insulin Sensitivity Check Index Is Better Correlated to Hyperinsulinemic Glucose Clamp than Other Fasting-Based Index of Insulin Sensitivity in Different Insulin-Resistant States. J Clin Endocrinol Metab 88(10): p. 4917-4923. [Crossref]

20. McClain DA, Lubas WA, Cooksey RC, Hazel M, Parker GJ et al. (2002) Altered glycan-dependent signaling induces insulin resistance and hyperleptinemia. Proc Natl Acad Sci U S A 99(16): p. 10695-10699. [Crossref]

21. Ge RL, Simonson TS, Cooksey RC, Tanna U, Qin G, et al. (2012) Metabolic insight into mechanisms of high-altitude adaptation in Tibetans. Mol Genet Metab 106: 244-247. [Crossref]

22. Nam H, Jones D, Cooksey RC, Gao Y, et al. (2016) Sink S Synergistic inhibitory effects of hypoxia and iron deficiency on hepatic glucose response in mouse liver. Diabetes [Crossref]

23. Wang W, Knovich MA, Coffman LG, Torti FM, Torti SV (2010) Serum ferritin: Past, present and future. Biochim Biophys Acta 1800: 760-769. [Crossref]

24. Kunutsor SK (2013) Ferritin levels and risk of type 2 diabetes mellitus: an updated systematic review and meta-analysis of prospective evidence. Diabetes Metab Res Rev 29(4): p. 308-318.

25. Orban E (2014) Association of iron indices and type 2 diabetes: a meta-analysis of observational studies. Diabetes Metab Res Rev 30(5): p. 372-394.

26. Fu S, Li F, Zhou J, Liu Z (2016) The Relationship Between Body Iron Status, Iron Intake And Gestational Diabetes: A Systematic Review and Meta-Analysis. Medicine 95(2): p. e2383. [Crossref]

27. McClain DA, Abraham D, Rogers J, Brady R, Gault P, et al. (2006) High prevalence of abnormal glucose homeostasis secondary to decreased insulin secretion in individuals with hereditary haemochromatosis. Diabetologia 49(7): p. 1661-1669. [Crossref]

28. Creighton Mitchell T, McClain DA (2014) Diabetes and hemochromatosis. Curr Diab Rep 14: 488. [Crossref]

29. Gordeuk VR, McLaren CE, Looker AC, Hasselblad V, Brittenham GM (1998) Distribution of Transferrin Saturations in the African-American Population. Blood 91(6): p. 2175. [Crossref]

30. Ashourpour M, Djalali M, Djazayery A, Eshraghian MR, Taghdir M, et al. (2010) Relationship between serum ferritin and inflammatory biomarkers with insulin resistance in a Persian population with type 2 diabetes and healthy people. Int $J$ Food Sci Nutr 61(3): p. 316-323. [Crossref]

31. Gordeuk VR, Reboussin DM, McLaren CE, Barton JC, Acton RT, et al. (2008) Serum ferritin concentrations and body iron stores in a multicenter, multiethnic primary-care population. Am J Hematol 83(8): p. 618-26. [Crossref]

32. Ryan JD, Armitage AE, Cobbold JF, Banerjee R, Borsani O, et al. (2017) Hepatic iron is the major determinant of serum ferritin in NAFLD patients. Liver Int [Crossref]

33. Valenti L, Fracanzani AL, Dongiovanni P, Bugianesi E, Marchesini G, et al. (2007) Iron depletion by phlebotomy improves insulin resistance in patients with nonalcoholic fatty liver disease and hyperferritinemia: evidence from a case-control study. Am J Gastroenterol 102(6): p. 1251-1258. [Crossref]

34. Beaton MD, Chakrabarti S, Adams PC (2014) Inflammation is not the cause of an elevated serum ferritin in non-alcoholic fatty liver disease. Ann Hepatol 13(3): p. 353-356. [Crossref] 
35. Woolcott OO, Castillo OA, Gutierrez C, Elashoff RM, Stefanovski D, et al. (2014) Inverse Association between Diabetes and Altitude: A Cross Sectional Study in the Adult Population of the United States. Obesity (Silver Spring, Md.) 22(9): p. 2080-2090. [Crossref]

36. American Diabetes Association (2009) Diagnosis and Classification of Diabetes Mellitus. Diabetes Care 32(Suppl 1): p. S62-S67.

37. McClain DA, Abuelgasim KA, Nouraie M, Salomon-Andonie J, Niu X, et al (2013) Decreased serum glucose and glycosylated hemoglobin levels in patients with Chuvash polycythemia: a role for HIF in glucose metabolism. $J$ Mol Med (Berl) 91(1): p. 59-67. [Crossref]

38. Weir MR, Klassen DK, Hoover N, Douglas FL (1989) Preliminary observations of the acute effects of selective serum thromboxane inhibition and angiotensin converting enzyme inhibition on blood pressure and renal hemodynamics in hypertensive humans. J Clin Pharmacol 29(12): p. 1108-1116. [Crossref]

\section{Citation:}

Zhenzhong Bai, Chengyu Zhao, Shou Liu, Renjie Feng, Sen Cui, Ri-li Ge, Donald McClain (2018) Higher Serum Ferritin in Tibetan and Han Populations with Diabetes Living on the Tibetan Plateau. Endocrinol Diabetes Metab J Volume 2(1): 1-7 